\title{
Electrocortical Effects of Acetaminophen during Emotional Picture Viewing, Cognitive Control, and Negative Feedback
}

\author{
Katie E. Garrison ${ }^{1}$ (D) - Julia B. McDonald ${ }^{2}$. Adrienne L. Crowell ${ }^{3} \cdot$ Nicholas J. Kelley $^{4} \cdot$ Brandon J. Schmeichel $^{5}$
}

Accepted: 7 January 2021 / Published online: 19 February 2021

(C) The Psychonomic Society, Inc. 2021

\begin{abstract}
Acetaminophen, the active ingredient in Tylenol, may have psychological effects, such as reducing social and emotional pain. The current study $(N=173)$ used electroencephalography (EEG) to extend past research on acetaminophen. Healthy undergraduate students $(64.7 \%$ women, age $M=18.15, S D=3.33)$ were randomly assigned to ingest $1,000 \mathrm{mg}$ of acetaminophen or placebo before completing emotional picture viewing $(n=143)$, a flanker task $(n=69)$, and a probabilistic learning task $(n=143)$ while EEG was recorded. (Sample sizes used for the analyses of each task differ from the total $N$ due to data loss.) We observed standard event-related potentials (ERPs), including emotion-modulated late positive potentials during picture viewing and feedback-related negativity during feedback on the probabilistic learning task. We also observed standard error-related and conflict-related ERPs in the flanker task but could not adequately assess acetaminophen's effect on flanker ERPs due to excessive data loss. Acetaminophen did not alter any of the ERPs, in contrast to predictions based on prior research. Exploratory analyses revealed that acetaminophen reduced the relationship between trait behavioral inhibition system sensitivity and emotionmodulated late positive potentials. Together these findings suggest that a standard dose of acetaminophen did not reliably alter neural indicators of emotional or feedback processing. Instead, preliminary findings from our study suggested that a more nuanced relationship may exist between acetaminophen and individual differences in emotional processing, although this latter finding calls for further replication.
\end{abstract}

Keywords Acetaminophen $\cdot$ Emotion $\cdot$ Cognitive control $\cdot$ Psychophysiology

Acetaminophen (paracetamol), the active ingredient in the popular over-the-counter drug Tylenol, is commonly used to relieve pain for a variety of physical ailments. An accumulating body of evidence has suggested that acetaminophen also has widespread psychological effects (DeWall et al., 2010; DeWall, Chester, \& White, 2015; Durso, Luttrell, \& Way, 2015; Randles, Heine, \& Santos, 2013; Randles, Kam,

Katie E. Garrison

garrison.katie.e@gmail.com

1 Department of Psychology, The University of Alabama, Tuscaloosa, AL 35401, USA

2 Department of Psychology, University of South Florida, Tampa, FL 33620, USA

3 Department of Psychology, Sacred Heart University, Fairfield, CT 06825, USA

4 Department of Psychology, University of Southampton, Southampton, UK

5 Texas A\&M University, College Station, TX 77843, USA
Heine, Inzlicht, \& Handy, 2016). The current study extended past research on acetaminophen by testing its effects on electrocortical measures of emotional processing, cognitive control, and responses to negative feedback.

\section{Overview of previous research}

One influential pair of experiments tested the effects of acetaminophen on the emotional pain of being socially excluded (DeWall et al., 2010). In the first experiment, participants took either acetaminophen or placebo for 3 weeks, and they supplied daily reports on experiences in their social relationships. Participants who took acetaminophen reported less intense hurt feelings compared with those who took a placebo, consistent with the idea that acetaminophen reduces not just physical but also psychological or emotional pain. In a second experiment, participants consumed either acetaminophen or placebo for 3 weeks before completing a laboratory-based game involving social exclusion while fMRI measured brain activity. The results revealed less activity in the dorsal anterior 
cingulate cortex (dACC) and anterior insula during the exclusion game among participants who took acetaminophen; $\mathrm{dACC}$ and anterior insula activation have been associated with the experience of social pain in prior research (Eisenberger, Lieberman, \& Williams, 2003). Additional work has found that just one dose of acetaminophen reduces self-reported empathy toward another's pain as described in hypothetical scenarios (Mischkowski, Crocker, \& Way, 2016; Mischkowski, Crocker, \& Way, 2019). This research supports the notion that acetaminophen blunts both self-reported and associated neural correlates of emotional or psychological pain.

Other research has built on the idea that acetaminophen reduces psychological pain by finding that an acute dose of acetaminophen reduces the use of psychological defenses in response to aversive events, such as thinking of one's own mortality (Randles et al., 2013). A dose of acetaminophen also has been observed to ease the discomfort or dissonance associated with making a difficult decision (DeWall et al., 2015). These findings suggest that acetaminophen may reduce not only emotional pain experiences but also psychological responses to aversive or threatening situations.

Another influential experiment found evidence to suggest that the effects of acetaminophen extend beyond psychological pain to blunt self-reported emotional reactivity more generally (Durso et al., 2015). Participants in this experiment ingested a one-time 1,000-mg dosage of either acetaminophen or placebo. Approximately 60 minutes later, allowing for the drug to metabolize, participants viewed and evaluated a series of emotionally charged images selected from the International Affective Picture System (IAPS; Lang, Bradley, \& Cuthbert, 2008). Images ranged from extremely pleasant to view (e.g., children playing, people laughing) to extremely unpleasant to view (e.g., mutilated body parts, gun pointed at viewer). Participants rated how positive or negative the photos were perceived. Then, they viewed the photos again and rated how emotionally aroused the images made them feel. Acetaminophen, compared with placebo, reduced emotional ratings and self-reported arousal to both positive and negative images, suggesting a general blunting effect of acetaminophen on emotional and evaluative processing.

Previous research has thus observed intriguing effects of acetaminophen on emotional responding and emotional pain. The principle purpose of the present research was to extend these findings to neural indices of emotional processing. The guiding question was: Can the emotion-blunting effects of acetaminophen be glimpsed in the electroencephalogram?

In addition to emotional processes, acetaminophen has been found to influence behavioral and neural measures of cognitive control. In one study participants ingested $1,000 \mathrm{mg}$ of acetaminophen or placebo before completing a Go/NoGo task, which involves the inhibition of prepotent responses (Randles et al., 2016). Acetaminophen increased errors of omission (e.g., misses) but not errors of commission (e.g., false alarms), and it reduced the amplitude of a neural signature of error processing, the error-positivity $(\mathrm{Pe})$. This event-related potential (ERP) is a sustained positivity in the electroencephalogram occurring approximately 180-300 ms following an erroneous response and is thought to index sconscious awareness of errors (Hajcak, McDonald, \& Simons, 2003; Nieuwenhuis, Ridderinkhof, Blom, Band, \& Kok, 2001). However, the error-related negativity (ERN), an earlier ERP component defined by a sharp negative deflection approximately 50-100 ms following an error, was not influenced by acetaminophen. This pattern suggested that acetaminophen influences downstream conscious evaluation of one's errors but not early recognition of error commission. Insofar as cognitive control and error processing are aversive (Dreisbach \& Fischer, 2012; Elkins-Brown, Saunders, \& Inzlicht, 2016; Hajcak \& Foti, 2008; Inzlicht, Bartholow, \& Hirsh, 2015; Spunt, Lieberman, Cohen, \& Eisenberger, 2012), this evidence on neural responses to errors is broadly consistent with the emotional blunting effects of acetaminophen reviewed above. We sought to replicate these findings conceptually and extend them by testing the hypothesis that acetaminophen reduces responding to error feedback on a probabilistic learning task.

\section{Overview of present research}

Psychological effects of acetaminophen could have major societal implications, given the popularity and accessibility of the drug. Continued efforts to replicate and extend the findings reviewed above are necessary before considering any recommendations for the general public or for public policy (e.g., warning labels or drug regulations; Ratner, Kaczmarek, \& Hong, 2018). Only a handful of studies exist in the published record, and many of them included self-report measures only or tested small samples of participants (a point we return to in the Discussion). The current study tested a relatively large sample of participants and used neural measures to extend past findings. We had three major goals: 1) to test the blunting effects of acetaminophen on the late positive potential (LPP), a well-validated electrophysiological measure of emotional processing; 2) to replicate past research on the effects of acetaminophen on cognitive control, particularly its electrophysiological components including the error-positivity $(\mathrm{Pe})$; and 3) a novel test of acetaminophen's effects on feedback processing during probabilistic learning.

We tested acetaminophen's effects on multiple outcome measures based on the assumption that once the drug metabolized, its effects would persist for the duration of the three tasks. Indeed, research has found that when ingested orally, acetaminophen reaches peak concentrations within 45-60 minutes. The analgesic effects can last approximately 4 hours 
with a half-life of 1.5-3 hours (Bertolini et al., 2006; Yoon, Babar, Choudhary, Kutner, \& Pyrsopoulos, 2016). Participants completed a passive picture-viewing task of emotionally arousing and neutral images while we measured electroencephalographic (EEG) activity. Previous research found that acetaminophen reduces self-reports of emotional experience, but self-reports are subject to a host of biasing influences, including perceived social appropriateness and demand characteristics. Neural measures are relatively impervious to these reporting biases. We analyzed an ERP component known as the LPP, which indexes the elaborated processing of behaviorally relevant stimuli and is amplified in response to motivationally salient or emotionally arousing stimuli (Cuthbert, Schupp, Bradley, Birbaumer, \& Lang, 2000; Foti, Hajcak, \& Dien, 2009; Hajcak, MacNamara, \& Olvet, 2010; Schupp et al., 2000; Schupp, Junghöfer, Weike, \& Hamm, 2004). More specifically, the LPP has been linked to the processing of emotional pictures, such that the LPP is larger in amplitude while viewing arousing positive and negative pictures relative to neutral or non-arousing pictures (Hajcak et al., 2010; Schupp et al., 2000). We predicted that acetaminophen would reduce LPP magnitudes to both negative and positive images based on evidence that acetaminophen blunts selfreported emotional reactivity (Durso et al., 2015).

Participants also completed a flanker task so that we could conceptually replicate the finding reported by Randles et al. (2016) reviewed above. However, due to a combination of technical and experimenter errors, a preponderance of participants' responses during the flanker task were coded as $0 \mathrm{~s}$ in the data set, which drastically reduced the number of usable trials remaining for statistical analysis and reduced the internal reliability of the flanker ERPs (Chronbach's alphas ranged from 0.569 to 0.901 ). We therefore opted to forego a thorough presentation and interpretation of the data afforded by this task and refer interested readers to the flanker-related results presented in supplemental online materials.

Last, participants completed a test of probabilistic learning that consisted of several blocks of an implicit learning task accompanied by positive and negative feedback. We assessed a neural index of feedback processing, the feedback-related negativity (FRN). This component is involved in performance-monitoring and is larger to negative feedback indicating errors or loss relative to positive feedback (Cavanagh, Bismark, Frank, \& Allen, 2011; Holroyd \& Coles, 2002; Schmid, Hackel, Jasperse, \& Amodio, 2018; Yeung, Holroyd, \& Cohen, 2004). The FRN and the ERN have been thought to reflect common neural processes (Frank, Woroch, \& Curran, 2005; Potts, Martin, Kamp, \& Donchin, 2011). We reasoned that receiving negative feedback, although essential for learning, is an aversive experience and ERPs associated with negative feedback therefore may be reduced by acetaminophen. More recent work has found that the feedback-related ERP may actually reflect reward-related neural activity to gains versus losses (Proudfit, 2015). If the FRN is sensitive to rewarding outcomes, then it still may be blunted by acetaminophen, because acetaminophen is thought to blunt responses to both negative and positive emotional events (Durso et al., 2015).

In addition to probing the main effects of acetaminophen on affective responding, cognitive control, and probabilistic learning, respectively, we also explored possible moderating effects of individual differences in emotional responding. Individual differences in sensitivity of the behavioral inhibition system (BIS) and behavioral activation system (BAS), in particular, have been found to relate to psychophysiological responding. For instance, individuals higher in BIS display larger LPPs to negative emotional images and larger ERNs following errors (Balconi, Falbo, \& Conte, 2012; Boksem, Tops, Kostermans, \& De Cremer, 2008; Boksem, Tops, Wester, Meijman, \& Lorist, 2006), whereas individuals higher in BAS have been found to display larger LPPs to positive images (Balconi et al., 2012) and larger Pe amplitudes following errors (Boksem et al., 2006; Boksem et al., 2008). Because BIS and BAS sensitivities are relevant for emotional and error processing, we thought that acetaminophen may operate differently as a function of individual differences in BIS and BAS sensitivities. We therefore incorporated BIS and BAS into our analyses.

\section{Method}

\section{Participants and design}

Healthy undergraduate students $(N=173)$ completed the experiment in exchange for credit toward a course requirement. Due to a computer error, gender and age data were only recorded for 34 participants (64.7\% women, age $M=18.15$, SD $=3.33$ ). We screened participants and excluded those who had consumed acetaminophen within the past 24 hours. This study was approved by the university's Institutional Review Board. Participants were randomly assigned to either the acetaminophen condition $(n=93)$ or placebo condition $(n=80)$; both the experimenter and the participant were blind to condition. Approximately 1 hour after taking the dose, participants completed three computer tasks (described below) while their brain activity was monitored using EEG.

\section{Procedures}

After providing informed consent participants received a brief description about the experiment. Participants were randomly assigned to ingest either $1,000 \mathrm{mg}$ of acetaminophen or placebo (cornstarch capsule) before EEG sensor placement. Sensor placement lasted approximately 45-60 minutes; on average participants began their first task 49.38 minutes $(S D=$ 
11.10) after ingestion. Acetaminophen is thought to reach peak concentrations within 45-60 minutes, with analgesic effects lasting about 4 hours - well beyond the length of the experiment (Bertolini et al., 2006; Smith, 2009; Yoon et al., 2016). During EEG hookup, participants completed a series of questionnaires, including behavioral inhibition system and behavioral activation system (BIS/BAS) sensitivity (Carver \& White, 1994), handedness (adapted from Oldfield, 1971), PANAS-X (Watson \& Clark, 1999), and impulsivity (Barratt, 1994) and provided basic demographic information. Please see the supplemental materials for bivariate correlations between the individual difference measures and the ERP measures. See Table 1 for descriptive information about the participants as a function of pill condition. ${ }^{1}$

After sensor placement, participants sat for a 4-minute resting recording alternating between eyes open and closed every minute. Participants then completed three computer tasks in a randomly determined order: a picture-viewing task, a flanker task, and a probabilistic learning task (see supplemental materials for flanker-related methods and results). We administered acetaminophen (or placebo) prior to EEG hookup so that participants would experience peak concentrations of acetaminophen during the tasks described below.

Picture-viewing task Participants passively viewed a series of emotional and neutral pictures on the computer screen. Pictures were presented using DMDX software. Participants viewed 19 positive, 19 neutral, and 19 negative pictures borrowed from the International Affective Picture System (IAPS; Lang et al., 2008). ${ }^{2}$ Positive pictures depicted exciting or fun activities. Neutral pictures featured ordinary objects and mundane scenes. Negative pictures include mainly scenes of violence or mutilation. The first four pictures were neutral practice trials and were not analyzed. Each trial consisted of a fixation cross that appeared onscreen for 3 seconds, followed by a picture for 6 seconds, and an intertrial interval of 8-12 seconds. The picture-viewing task lasted 22 minutes.

Probabilistic learning task The probabilistic learning task (PLT) was borrowed from previous research (Cavanagh et al., 2011; Frank et al., 2005; Gründler, Cavanagh, Figueroa, Frank, \& Allen, 2009). The PLT consisted of four training blocks followed by one test block. During the training phase, participants saw two Japanese characters onscreen (e.g., あ も) simultaneously and

\footnotetext{
${ }^{1}$ Because there were group differences in trait impulsivity, we assessed whether trait impulsivity moderated the effect of pill condition. Trait impulsivity did not interact with pill condition to influence any of the ERP measures, $p s>0.405$.

2 The following IAPS photos were used. Negative valence: 1052, 1205, 1270, 1300, 2811, 3000, 3022, 3071, 3130, 3150, 3250, 3400, 3550, 6230, 6550, $6560,7380,9300,9405$. Neutral valence: $2190,2393,2394,2397,2506$, 2516, 2516, 2850, 5534, 7000, 7009, 7025, 7035, 7053, 7100, 7161, 7180, 7185, 7236. Positive valence: 4608, 4651, 4656, 4658, 4659, 4670, 4681, $4695,5621,7200,7260,7350,7390,7460,7470,8031,8161,8186,8260$
}

were instructed to choose one. They were told that some symbols would have a higher chance of being correct, but they would not know which one. Their goal was to try and pick the one that was correct. The task included three pairs of stimuli, and each pair was associated with a specific probability of getting "correct" or "incorrect" feedback. The probabilities of getting "correct" feedback for each of the three stimuli pairs was 80/20, 70/30, and 60/ 40. As participants completed the training phase, they presumably learned which stimulus of each pair had the higher probability of returning "correct" feedback.

Trials began with an intertrial interval ranging from 300-700 $\mathrm{ms}$, followed by the pair of stimuli for 2,000 ms. Participants made a choice by pressing the left or right shift key corresponding to the left or right symbol, respectively. The stimuli disappeared from the screen after participants made a choice, and "Correct" or "Incorrect" feedback appeared for $500 \mathrm{~ms}$. If participants did not choose within $2,000 \mathrm{~ms}$, the feedback "No Response Detected" was presented for $500 \mathrm{~ms}$, followed by the next trial. Participant completed four blocks of the training phase.

At the end of the experiment, participants reported whether they believed they had received acetaminophen or placebo and how sure they were of their answer. Unexpectedly, participants' guesses about which pill they ingested differed between conditions. In the placebo condition, $54.3 \%$ of participants guessed they took the placebo and $45.7 \%$ guessed they took acetaminophen, whereas in the acetaminophen condition $63.3 \%$ of participants guessed they took acetaminophen and $37.7 \%$ of participants guessed they took placebo, $\chi^{2}(1)=4.68, p=0.030$. These results suggested that participants had some insight into the type of drug they had received. However, participants expressed only modest certainty in their guesses (percent certainty $M=55.23 \%, S D=24.79$ ) and level of certainly did not differ between conditions, $t$ (169) = $0.87, p=0.387$. A variable capturing whether participants guessed the pill condition correctly is included as a statistical covariate in the analyses reported below. Lastly, participants were debriefed about the purpose of the study and dismissed.

\section{Psychophysiological recording and quantification}

EEG was recorded with 59 tin electrodes in a stretch-lycra electrode cap using Neuroscan software for impedance checking and data collection. EEG signals were amplified with Neuroscan SynAmps2 (El Paso, TX), bandpass filtered $(0.05-100 \mathrm{~Hz})$, notch filtered $(60 \mathrm{~Hz})$ and digitized at $500 \mathrm{~Hz}$. Eye movements were recorded from an electrode at FP2 (10-20 placement system). Data were visually inspected and portions of the data that contained artifacts (e.g., horizontal eye movements, muscle movements) were first removed by hand. Then, a regressionbased eye movement correction was applied to correct vertical eye movements (Semlitsch, Anderer, Schuster, \& Presslich, 1986), after which the data were again visually inspected to ensure proper correction. Offline, data were re-referenced to the average earlobes. 
Table 1 Characteristics of participants as a function of pill condition

\begin{tabular}{lllr}
\hline & Placebo $M(S D)$ & Acetaminophen $M(S D)$ & Sig. \\
\hline BIS $(\alpha=0.748)$ & $2.92(0.49)$ & $2.96(0.44)$ & 0.567 \\
BAS $(\alpha=0.800)$ & $3.06(0.37)$ & $3.02(0.35)$ & 0.473 \\
Trait Positive Affect $(\alpha=0.869)$ & $3.23(0.67)$ & $3.39(0.61)$ & 0.113 \\
Trait Negative Affect $(\alpha=0.841)$ & $1.83(0.56)$ & $1.88(0.55)$ & 0.604 \\
Trait Impulsivity $(\alpha=0.783)$ & $2.22(0.30)$ & $2.13(0.28)$ & 0.050 \\
Handedness $(\alpha=0.767)$ & $2.76(0.27)$ & $2.76(0.28)$ & 0.916 \\
\hline
\end{tabular}

Picture-viewing task ERPs EEG data were epoched from $100 \mathrm{~ms}$ before to $1,000 \mathrm{~ms}$ after picture onset, low pass filtered $(16 \mathrm{~Hz}$, at $12 \mathrm{~dB})$, and baseline corrected using $100 \mathrm{~ms}$ of prestimulus activity. Low-pass filtering is typical for the low-frequency LPP wave and has been used in prior research (Finley, Crowell, \& Schmeichel, 2018; Garrison, Crowell, Finley, \& Schmeichel, 2017; Hajcak \& Nieuwenhuis, 2006). The data were then averaged for each picture type separately (positive, negative, and neutral). The LPP was quantified as the mean amplitude 500-1,000 ms following the onset of the image at electrode site Pz, where the LPP magnitude was maximal. We assessed internal consistency of the ERP measures by using within-subjects condition-averaged ERPs (e.g., trial type) to serve as "items" for Cronbach's alpha (Thigpen, Kappenman, \& Keil, 2017). The Cronbach's alpha for LPP averages at $\mathrm{Pz}$ to neutral, negative, and positive images was high, $\alpha=0.811$.

Probabilistic learning task ERPs EEG data was epoched from $200 \mathrm{~ms}$ before to $500 \mathrm{~ms}$ after feedback, band pass filtered (1$15 \mathrm{~Hz}$, at $24 \mathrm{~dB}$ ), and baseline corrected using $200 \mathrm{~ms}$ of prestimulus activity. There is a great deal of heterogeneity in band pass filter settings across prior studies, but we elected to use the same settings used by the researchers who pioneered the probabilistic learning task (Cavanagh et al., 2011; Gründler et al., 2009). Data were averaged for correct and incorrect feedback trials separately. The FRN was defined as the peak-to-trough difference between the maximum negative peak within a window of 190-300 ms following feedback and the preceding positive peak at electrode site $\mathrm{FCz}$ (Cavanagh et al., 2011; Schmid et al., 2018). ${ }^{3}$ We included both the FRN peak and trough values (both of which went into estimation of the FRN) for the correct and incorrect averages at FCz (four

\footnotetext{
${ }^{3}$ We also calculated the reward-positivity (RewP) component by subtracting the incorrect feedback waveform from the correct feedback waveform and creating a difference wave (as is commonly done). We then extracted mean amplitudes for the RewP at electrode FCz in the time window of 200-350 ms based on past research and an inspection of the grand average waveform. Then we assessed the main effect of pill condition on this component and found no significant effect, $t(142)=-0.64, p=.524$. Further, a linear regression testing the extent to which trait BAS moderates of the effect of pill condition revealed a non-significant effect of BAS, $B=0.359, S E=0.42, t=-0.66, p=0.512$, and no BAS x Pill Condition interaction, $B=1.25, S E=0.84, t=1.48, p=0.141$.
}

items) to calculate Cronbach's alpha, and the internal consistency was high, $\alpha=0.803$.

\section{Data analysis plan}

We analyzed the results from each task separately. We conducted a Pill Condition $\times$ Trial Type mixed-factorial analysis of variance (ANOVA) on each ERP measure. A significant effect of trial type confirms the presence of the ERPs of interest. For the picture task, we assessed LPP magnitudes as a function of picture type. For the probabilistic learning task, we assessed FRN amplitudes as a function of feedback type. For the Flanker task, we assessed N2 magnitudes as a function of trial congruency and ERN and Pe magnitudes as a function of trial accuracy (see SOM). Then, we tested the effects of pill condition on the ERPs of interest.

Following the primary analyses, we added task order and condition guess accuracy (i.e., whether or not participants correctly guessed their pill condition) as covariates. It also was possible that task order and guess accuracy moderated the effects of acetaminophen, but this study was not intended to test such moderating effects and was not well-powered to produce robust parameter estimates for such analyses; the covariate approach does not sacrifice statistical power to the same degree. We therefore report exploratory analyses treating task order and condition guesses as moderator variables in the SOM, and below we report the primary analyses with these variables treated as statistical covariates.

Last, we tested whether the effects of acetaminophen were moderated by trait BIS and BAS because of their relevance for psychophysiological responding (Balconi et al., 2012; Boksem et al., 2006; Boksem et al., 2008).

\section{Results}

\section{Picture-viewing task}

Thirty participants were excluded from the picture-viewing task analysis due to missing or unusable EEG data, leaving a final sample of 143 students (Placebo $n=62$, Acetaminophen $n=81$ ). A 2 (Pill Condition: acetaminophen vs. placebo) $\times 3$ 
(Picture Type: positive, negative, neutral) mixed-factorial ANOVA on LPP magnitudes found a main effect of picture type, $F(2,282)=110.78, p<0.001, \eta_{\mathrm{p}}{ }^{2}=0.440$. Consistent with previous research (Cuthbert et al., 2000; Hajcak et al., 2010; Schupp et al., 2004), LPP magnitudes were larger to negative images $(M=10.87, S D=6.69)$ compared with positive images $(M=9.70, S D=6.43), t(142)=2.58, p=0.011, d$ $=0.22$, and larger to positive images compared with neutral images $(M=4.08, S D=5.87), t(142)=11.88, p<0.001, d=$ 0.99 . See Fig. 1 for a depiction of LPP magnitudes by picture type.

However, we found no main effect of pill condition, $F$ (1, 141) $=0.63, p=0.430, \eta_{\mathrm{p}}{ }^{2}=0.004$, and no interaction between pill condition and picture type, $F(2,282)=0.10, p=$ $0.908, \eta_{\mathrm{p}}{ }^{2}=0.001$. LPPs were similar in magnitude in the acetaminophen condition $(M=5.34, S D=0.59)$ versus the placebo condition $(M=5.49, S D=0.70), d=0.23$. This result does not support the hypothesis that acetaminophen blunts emotional processing. We conducted a sensitivity analysis to determine whether we had sufficient statistical power to detect the expected effects. Based on our sample size of 143 participants and an alpha error probability of 5\%, we had $80 \%$ power to detect an effect size for the Picture Type $\times$ Pill Condition interaction of $\eta_{\mathrm{p}}{ }^{2}=0.009$, and we observed a partial eta squared of $\eta_{\mathrm{p}}{ }^{2}=0.001$. Additionally, we had $80 \%$ power to detect a between-subjects effect of pill condition of $d=0.48$, and we observed an effect of $d=0.23$. Therefore, although our sample size was larger than most ERP studies, we may have been underpowered to detect small effects of acetaminophen.

Task order Participants completed three different tasks over the course of approximately 1.5 hours. We did not anticipate that task order would alter the effects of acetaminophen, because acetaminophen should be at peak concentrations during the entire experimental session (Bertolini et al., 2006; Yoon et al., 2016). Nevertheless, to statistically control for any effect of task order, we repeated the LPP analyses above with task order (whether the picture task was $1^{\text {st }}, 2^{\text {nd }}$, or $3^{\text {rd }}$ ) as a covariate and found that the statistical significance of the results remained unchanged: main effect of picture type, $F(2,280)$ $=17.62, p<0.001, \eta_{\mathrm{p}}{ }^{2}=0.112$, and pill condition main effect or interaction with picture type, $p \mathrm{~s}>0.436$.

Guess accuracy Next, we repeated the analyses controlling for guess accuracy. When we added as a covariate whether participants' guesses about which pill condition they were in were correct $(0)$ or not (1), the main effect of picture type on LPPs remained significant, $F(2,280)=36.17, p<0.001, \eta_{\mathrm{p}}{ }^{2}$ $=0.205$, the main effect of pill condition remained nonsignificant, $F(1,140)=1.11, p=0.294, \eta_{\mathrm{p}}{ }^{2}=0.008$, and the Picture Type $\times$ Pill Condition interaction remained nonsignificant, $F$ $(2,280)=0.19, p=0.831, \eta_{\mathrm{p}}{ }^{2}=0.001$.
Moderation by BIS and BAS Last, we explored the moderating effects of trait BIS and trait BAS on LPP magnitudes. These individual difference measures have been found to predict enhanced LPPs to emotional images (Balconi et al., 2012; Finley et al., 2018). If acetaminophen affects emotional processing, then it may act differently as a function of trait BIS and BAS. First, we ran a regression model predicting LPP magnitudes during emotional images (positive and negative), controlling for LPPs to neutral images, as a function of trait BIS (centered), pill condition, and their interaction. ${ }^{4} \mathrm{We}$ found that BIS and pill condition interacted to predict LPPs during emotional images, $B=-3.60, S E=1.70, t=2.12, p=0.036$, $95 \%$ confidence interval $(\mathrm{CI})[-6.95,-0.24]$.

To unpack the significant interaction term, we examined the correlations between BIS and LPP magnitudes in the acetaminophen and placebo conditions separately. In the placebo condition, we replicated past research in finding a positive correlation between BIS and LPP magnitudes to emotional images, $r(61)=0.38, p=0.003$. This relationship was reduced to nonsignificance in the acetaminophen condition, $r$ (74) $=-0.06, p=0.619$, and a Fisher's $Z$ test confirmed a significant difference between the two correlations, $Z=2.67$, $p=0.008$. The main effect of BIS on LPPs to emotional images also was significant, $B=1.91, S E=0.85, t=2.24, p$ $=0.027,95 \%$ CI $0.22,3.60]$. See Fig. 2 for a depiction of the relationship between BIS and LPPs to emotional images as a function of pill condition.

When we assessed the effects of BAS, neither the interaction between BAS and pill condition, $B=-0.60, S E=2.33, t=$ $-0.26, p=0.798,95 \%$ CI $[-5.20,4.01]$, nor the main effect of BAS, $B=-1.72, S E=1.16, t=-1.49, p=0.138,95 \% \mathrm{CI}$ $[-4.01,0.56]$, on emotional LPPs were significant. ${ }^{5}$

In sum, the LPP was modulated by picture type as in previous research-larger in magnitude for emotional compared with neutral images. Contrary to predictions, a dose of acetaminophen had no main effect on LPP magnitudes, but we discovered that acetaminophen broke the link between BIS and LPPs during emotional images.

\section{Probabilistic learning task}

After removing cases with missing or unusable EEG data for this task, we had a final sample of 143 students for analysis (Placebo $n=63$, Acetaminophen $n=80$ ). The FRN was

\footnotetext{
${ }^{4}$ Results were virtually identical when not controlling for neutral LPPs: BIS $\times$ Pill on emotional LPPs, $B=-5.01, S E=2.11, t=-2.39, p=0.018,95 \% \mathrm{CI}$ $[-9.23,-0.87]$.

${ }^{5}$ We also conducted regressions predicting LPPs to negative and positive images separately. The BIS $\times$ Pill Condition interaction was significant for LPPs during negative images, $B=-5.10, S E=2.03, t=2.52, p=0.013$, but not positive images, $B=-2.09, S E=1.91, t=1.09, p=0.276$. The BAS $\times$ Pill Condition interaction was nonsignificant for LPPs during both negative images, $B=-1.51, S E=2.76, t=0.55, p=0.586$, and positive images, $B=0.32$, $S E=2.61, t=0.12, p=0.903$.
} 


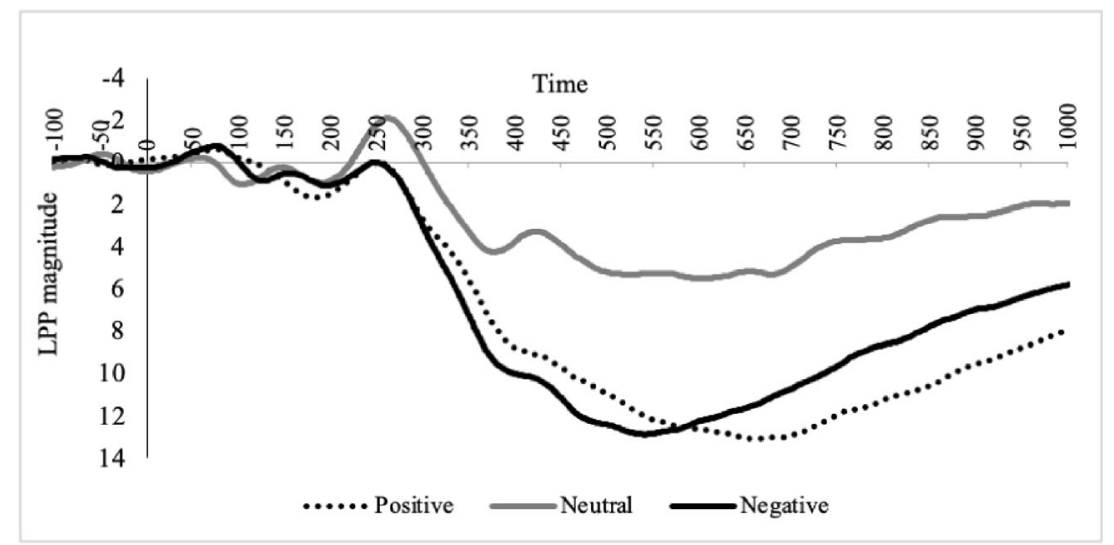

Fig. 1 Effect of Picture Type on LPP Magnitudes $(\mu \mathrm{V})$ at electrode Pz across time (ms)

defined as the peak-to-trough difference between the maximum negative peak within a window of 190-300 ms following feedback and the preceding positive peak at electrode. Because the values of this magnitude represent a difference score, greater numbers (more positive) indicate a larger peak. A 2 (Pill Condition: acetaminophen vs. placebo) $\times 2$ (Feedback: correct vs. incorrect) mixed-factorial ANOVA on FRN magnitudes found a main effect of feedback, $F$ (1, $141)=171.20, p<0.001, \eta_{\mathrm{p}}{ }^{2}=0.548$, such that FRNs were larger to incorrect feedback $(M=4.07, S D=2.49)$ versus correct feedback $(M=1.34, S D=1.71)$. See Fig. 3 for a depiction of FRN magnitudes by feedback type. Contrary to predictions, the main effect of pill condition was non-significant, $F(1,141)=0.01, p=0.928, \eta_{\mathrm{p}}{ }^{2}<0.001$, and the Pill Condition $\times$ Feedback Type interaction was also non-significant, $F(1,141)=1.18, p=0.280, \eta_{\mathrm{p}}{ }^{2}=0.008$. FRN magnitudes did not differ in the acetaminophen condition $(M=4.14$, $S D=2.40)$ versus the placebo condition $(M=3.98, S D=$ $2.56), d=0.07$. The effect of pill condition remained nonsignificant when task order was added as a covariate, $F(1,140)=$ $0.01, p=0.927$, and when guess accuracy was added as a covariate, $F(1,140)=0.02, p=0.898$. We again conducted a sensitivity analysis to assess our statistical power. Based on an alpha error probability of 5\% and a sample size of 143 participants, we had $80 \%$ power to detect an effect size for the Trial Type $\times$ Pill Condition interact of $\eta_{\mathrm{p}}{ }^{2}=0.018$, and we observed a partial eta squared of $\eta_{\mathrm{p}}{ }^{2}=0.008$. We had $80 \%$ power to detect a between-subjects effect of pill condition of $d$ $=0.47$, and we observed an effect of $d=0.07$. Therefore, if acetaminophen produced small but reliable effects, then we may have missed them.

To parallel the LPP results reported above, we also explored possible moderation of acetaminophen's effects on the FRN as a function of trait BIS and trait BAS. Neither BIS, $B=0.96, S E=0.91, t=1.05, p=0.295,95 \% \mathrm{CI}$ $[-0.84,2.75]$, nor BAS, $B=-0.04, S E=1.24, t=0.04, p=$ $0.971,95 \%$ CI $[-2.50,2.41]$, moderated the effects of acetaminophen on the FRN.

\section{Discussion}

The current research sought to extend past research on the psychological effects of acetaminophen. This study was not a direct replication of past work, but it was similar to and inspired by previous work on the effects of acetaminophen on emotional and error processing. The main goal was to extend research on acetaminophen's effects beyond selfreport outcomes to neural measures. We administered acetaminophen (versus placebo) to a relatively large sample of participants before assessing electrocortical activity during emotional picture viewing, cognitive control, and probabilistic learning. Contrary to predictions, acetaminophen had no significant main effects on any of the outcome variables. Acetaminophen did not blunt the LPPs during emotional picture viewing, but acetaminophen did interact with trait BIS to predict LPP magnitudes, as described below. Acetaminophen did not influence FRN magnitudes to incorrect feedback during probabilistic learning. Acetaminophen also did not influence N2 magnitudes to incongruent (conflicting) flanker trials, nor did it alter ERN or Pe magnitudes following errors on the flanker task (see

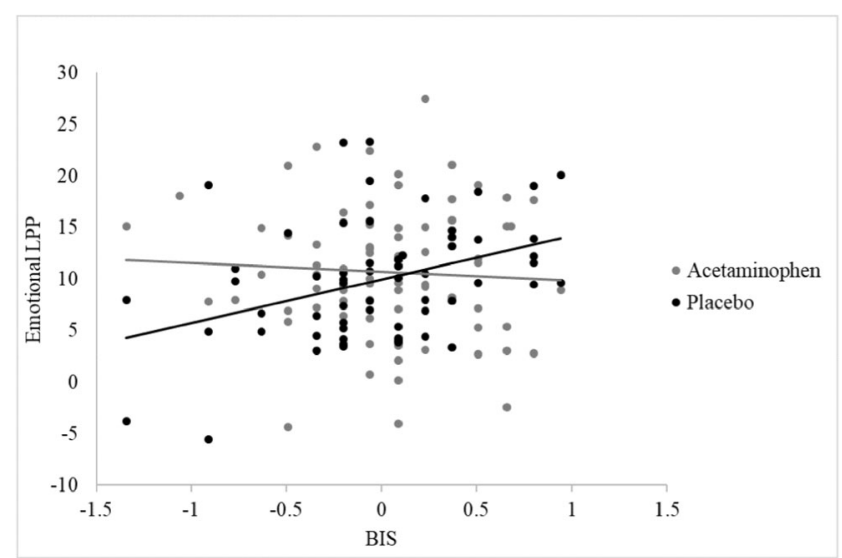

Fig. 2 Relationship between BIS and LPP Magnitudes during Emotional Images by Pill Condition 


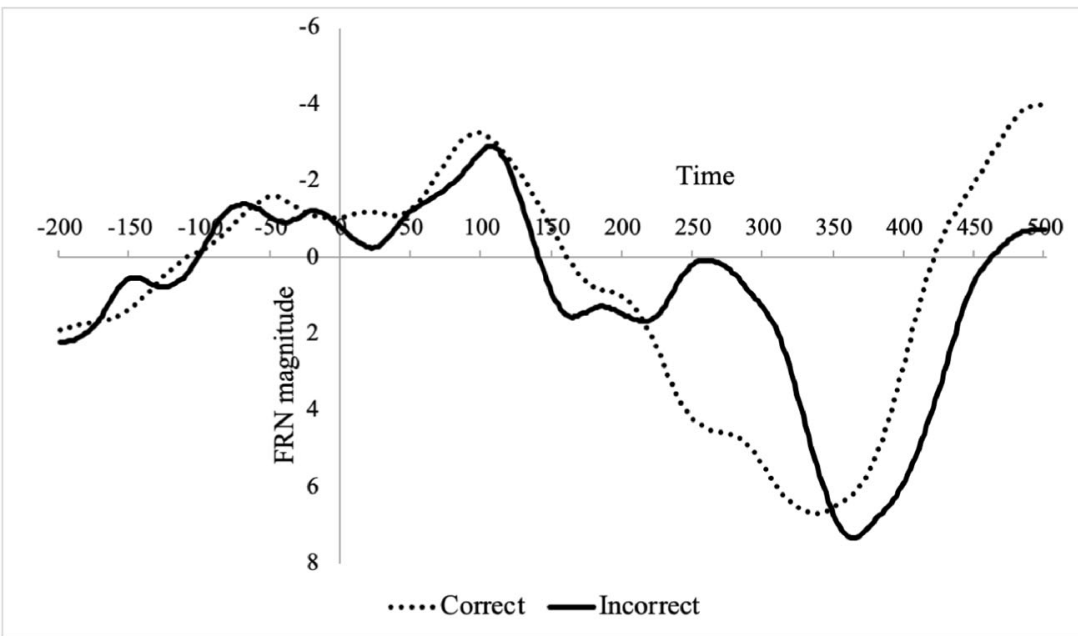

Fig. 3 Effect of Feedback Type on FRN Magnitudes ( $\mu \mathrm{V}$ ) at electrode $\mathrm{FCz}$ across time (ms)

supplemental materials for flanker results). In sum, we did not observe effects of acetaminophen that past research led us to expect.

Previous research found that acetaminophen reduces social or emotional pain. For instance, acetaminophen has been found to dampen self-reported distress and neural activity to social exclusion (DeWall et al., 2010), reduce empathy toward another's social or physical pain (Mischkowski et al., 2016; Mischkowski et al., 2019), and reduce self-reported emotional arousal and evaluations of emotional images (Durso et al., 2015). In the current study, we used the LPP as a neural marker of emotional processing based on evidence of enhanced LPPs to emotionally salient and highly arousing stimuli (Cuthbert et al., 2000; Foti et al., 2009; Schupp et al., 2000; Schupp et al., 2004). If acetaminophen blunts emotional processing, then why did acetaminophen fail to dampen neural correlates of emotional processing in the current study?

Acetaminophen may dampen specifically the evaluative components of social and emotional processing, for instance judgements of emotional events or decisions propelled by cognitive dissonance (Ratner et al., 2018). Perhaps acetaminophen did not alter the LPP, because this component reflects more downstream elaborative processes following initial stimulus evaluation. A recent study found that acetaminophen also did not reduce the LPP in the context of on-task versus off-task attention (Mutti et al., 2019), suggesting (as do the current results) that the LPP as a neural correlate of motivational salience and emotional arousal is relatively unaffected by a standard dosage of acetaminophen.

Another possibility is that acetaminophen blunts emotional processing by acting on brain regions other than those that underlie the LPP. The LPP is thought to index elaborated processing of motivationally or emotionally salient stimuli, and the neural generators of the LPP include areas of the visual cortex and subcortical emotionrelated processing regions, such as the amygdala (Liu, Huang, McGinnis-Deweese, Keil, \& Ding, 2012; Sabatinelli, Keil, Frank, \& Lang, 2013; Sabatinelli, Lang, Keil, \& Bradley, 2006). The mechanisms by which acetaminophen reduces pain are not well specified, however the consensus appears to be that acetaminophen acts via diverse mechanisms in the central nervous system (Smith, 2009; Toussaint et al., 2010). Acetaminophen's effects on physical pain correspond to reductions in brain activity in areas typically associated with pain processing such as the prefrontal cortices, ACC, insula, and thalamus (Pickering et al., 2015). The dACC and insula also are involved in the processing of social or emotional pain (Eisenberger et al., 2003). Research has found that acetaminophen blunts activity in these regions during social exclusion (Dewall et al., 2010). Although the physiological mechanisms of acetaminophen's analgesic effects are uncertain and likely multifaceted, the downstream impact on activity in the dACC and insula may be partially responsible for acetaminophen's psychological effects - and such effects may not be reflected in the LPP.

It is possible that the expected results did not emerge in the current study due to a lack of statistical power. One main contributor to statistical power is sample size. Previous research observed an emotional blunting effect of acetaminophen testing between 25 to 50 participants per experimental condition (DeWall et al., 2010; Durso et al., 2015; Mischkowski et al., 2016). The current study tested an even larger sample of participants. Specifically, the current study included 62 participants in the placebo condition and 81 participants in the acetaminophen condition (from the picture viewing LPP analyses). Yet, we still did not observe emotional blunting effects on the LPP. A sensitivity analysis on the Pill Condition $\times$ Trial 
Type interaction and the main effect of pill condition revealed that we were generally underpowered to detect small effects. Another consideration is the strength or potency of the experimental manipulation. We followed prior studies and administered 1,000 $\mathrm{mg}$ of acetaminophen; this is the dose at which acetaminophen reaches ceiling effects in human participants (Bertolini et al., 2006), so the efficacy of the manipulation is unlikely to account for the seemingly different results. Still, it is possible that unaccounted factors such as time since last meal could explain the lack of acetaminophen effects in the current study. We did not track time since last meal or instruct participants not to eat immediately before the study, although some prior studies have done so (Dewall et al., 2015; Mischkowski et al., 2016; Mischkowski et al., 2019).

The nonsignificant effects of acetaminophen on LPP magnitudes in the current study, in conjunction with past findings of blunted emotional arousal as assessed by selfreports, suggest that acetaminophen exerts a more noticeable influence on systems serving subjective experience relative to neural indices motivational salience (as indexed by the LPP). Future research should seek to identify the neural mechanisms by which acetaminophen reduces psychological pain and blunts emotional reactivity. This research would benefit from additional understanding of the neural mechanisms undergirding subjective emotional experience and the relationship between subjective experience and the LPP (which do not cohere, at least in some populations; Ellis, Schroder, Patrick, \& Moser, 2017). Presumably the effects of acetaminophen on emotional responding can be glimpsed in the brain, even though they do not appear to manifest in the LPP during emotional picture viewing.

Although a dose of acetaminophen did not blunt LPP magnitudes in general, it did interact with trait BIS to influence the LPP. Trait BIS sensitivity typically exhibits a positive correlation with the LPP in response to emotional stimuli (Balconi et al., 2012; Finley et al., 2018), consistent with the notion that individuals higher in BIS are more sensitive and responsive to emotional stimuli compared with those lower in BIS. We replicated this relationship in the placebo condition in the current study, but the link between BIS and LPP magnitudes was reduced to non-significance among participants who had taken acetaminophen. Acetaminophen thus modulated the association between BIS and neural responding to emotional stimuli. One implication of this pattern may be that acetaminophen alters the behavioral inhibition system, such that it is no longer reliably associated with enhanced reactivity to emotionally evocative stimuli. The interaction between acetaminophen consumption and BIS suggests that there may be meaningful individual differences in the ways in which acetaminophen impacts psychological processes. However, given that this finding was exploratory in nature, future research is needed to replicate this result.

Past research suggested that error processing, conflict detection, and negative feedback are aversive (Dreisbach \& Fischer, 2012; Elkins-Brown et al., 2016; Hajcak \& Foti, 2008; Inzlicht et al., 2015; Spunt et al., 2012), so we predicted that acetaminophen would dampen neural responses related to them much as acetaminophen dampens emotional pain. Furthermore, the ERN and FRN components following errors and negative feedback, respectively, are thought to be generated in the ACC, which may be one of the areas influenced by acetaminophen (Dewall et al., 2010; Pickering et al., 2015). However, we found no evidence that acetaminophen altered ERPs associated with those processes.

One shortcoming of the current work is the lack of selfreport measures of participants' emotional experiences. Past research found effects of acetaminophen on self-reported responses to a variety of events (e.g., emotional events, difficult decisions, existential threat), but the evidence that acetaminophen alters neural or psychophysiological responses is limited, and we found little evidence of acetaminophen effects in the EEG. It is possible that, relative to the emotional events studied in previous research, the emotional events from the current study (i.e., viewing images, receiving error feedback) were not sufficiently aversive to elicit effects of acetaminophen. Furthermore, many participants accurately guessed which pill (acetaminophen or placebo) they had taken, suggesting they may have had some subjective awareness of which drug they received. However, those guesses were of only modest certainty. When we statistically controlled for whether participants guessed correctly which substance (acetaminophen or placebo) they had consumed, the obtained EEG results were unchanged. When we treated guess accuracy as a moderator variable, some unexpected patterns emerged. Specifically, as described in the online supplemental materials, the effect of the pill on LPPs during the picture task was significant when participants were incorrect about their pill condition, such that LPPs were larger in the acetaminophen condition versus the placebo condition, but there was no effect of acetaminophen on LPPs when participants correctly guessed their pill condition. We wish not to overinterpret that finding; the analysis was unplanned, and the inclusion of a between-subjects moderator variable in the analysis dramatically reduced statistical power. Hence, these analyses were exploratory and underpowered and would need to be replicated.

\section{Conclusions}

The current work represents a step toward more robust research examining the effects of acetaminophen on neural 
and psychological processes. Future work should continue this effort, focusing on replicating prior findings with large samples. Given the widespread use of acetaminophen, rigorous research examining its potential psychological effects is essential and could have major implications for the public at large, although the results from the current study suggest that some robust neural correlates of emotional processing are relatively unaffected by a standard dosage of acetaminophen.

Supplementary Information The online version contains supplementary material available at https://doi.org/10.3758/s13415-021-00866-0.

\section{Declarations}

Conflict of interest On behalf of all authors, the corresponding author states that there is no conflict of interest.

\section{References}

Balconi, M., Falbo, L., \& Conte, V.A. (2012). BIS and BAS correlates with psychophysiological and cortical response systems during aversive and appetitive emotional stimuli processing. Motivation and Emotion, 36, 218-231.

Barratt, E.S. (1994). Impulsiveness and aggression. In J. Monahan \& H.J. Stedman (Eds), Violence and mental disorder: Developments in risk assessment. Chicago, University of Chicago Press.

Bertolini, A., Ferrari, A., Ottani, A., Guerzoni, S., Tacchi, R., \& Leone, S. (2006). Paracetamol: New vistas of an old drug. CNS Drug Reviews, $12,250-275$.

Boksem, M. A., Tops, M., Kostermans, E., \& De Cremer, D. (2008). Sensitivity to punishment and reward omission: evidence from error-related ERP components. Biological Psychology, 79, 185-192.

Boksem, M. A., Tops, M., Wester, A. E., Meijman, T. F., \& Lorist, M. M. (2006). Error-related ERP components and individual differences in punishment and reward sensitivity. Brain Research, 1101, 92-101.

Carver, C. S., White, T. I. (1994). Behavioral inhibition, behavioral activation and affective responses to impending reward and punishment: The BIS/ BAS scales. Journal of Personality and Social Psychology, 67, 319-333.

Cavanagh, J. F., Bismark, A., Frank, M. J., \& Allen, J. J. (2011). Larger error signals in major depression are associated with better avoidance learning. Frontiers in Psychology, 2, 331. https://doi.org/10. 3389/fpsyg.2011.00331

Cuthbert, B. N., Schupp, H. T., Bradley, M. M., Birbaumer, N., \& Lang, P. J. (2000). Brain potentials in affective picture processing: covariation with autonomic arousal and affective report. Biological Psychology, 52, 95-111.

DeWall, C. N., MacDonald G., Webster G. D., Masten C. L., Baumeister R. F., Powell C., \& Eisenberger N. I. (2010). Acetaminophen reduces social pain: Behavioral and neural evidence. Psychological Science, 21, 931-937.

DeWall, C. N., Chester, D. S., \& White, D. S. (2015). Can acetaminophen reduce the pain of decision-making? Journal of Experimental Social Psychology, 56, 117-120.

Dreisbach, G., \& Fischer, R. (2012). Conflicts as aversive signals. Brain and Cognition, 78, 94-98.

Durso G. R. O., Luttrell A., Way B. M., (2015). Over-the-counter relief from pains and pleasures alike: Acetaminophen blunts evaluation sensitivity to both negative and positive stimuli. Psychological Science, 26, 750-758.

Eisenberger, N. I., Lieberman, M. D., \& Williams, K. D. (2003). Does rejection hurt? An fMRI study of social exclusion. Science, 302, 290-292.

Elkins-Brown, N., Saunders, B., \& Inzlicht, M. (2016). Error-related electromyographic activity over the corrugator supercilii is associated with neural performance monitoring. Psychophysiology, 53, 159-170.

Ellis, J. D., Schroder, H. S., Patrick, C. J., \& Moser, J. S. (2017). Emotional reactivity and regulation in individuals with psychopathic traits: Evidence for a disconnect between neurophysiology and selfreport. Psychophysiology, 54, 1574-1585.

Finley, A. J., Crowell, A. L., \& Schmeichel, B. J. (2018). Self-affirmation enhances processing of negative stimuli among threat-prone individuals. Social Cognitive and Affective Neuroscience, 13, 569-577.

Foti, D., Hajcak, G., \& Dien, J. (2009). Differentiating neural responses to emotional pictures: Evidence from temporal-spatial PCA. Psychophysiology, 46, 521-530.

Frank, M. J., Woroch, B. S., \& Curran, T. (2005). Error-related negativity predicts reinforcement learning and conflict biases. Neuron, 47, 495-501.

Garrison, K. E., Crowell, A. L., Finley, A. J., \& Schmeichel, B. J. (2017). Effects of prior mental effort on picture processing: An ERP investigation. Psychophysiology, 54, 1714-1725.

Gründler, T. O., Cavanagh, J. F., Figueroa, C. M., Frank, M. J., \& Allen, J. J. (2009). Task-related dissociation in ERN amplitude as a function of obsessive-compulsive symptoms. Neuropsychologia, 47, 1978-1987.

Hajcak, G., \& Foti, D. (2008). Errors are aversive: Defensive motivation and the error-related negativity. Psychological Science, 19, 103-108.

Hajcak, G., MacNamara, A., \& Olvet, D. M. (2010). Event-related potentials, emotion, and emotion regulation: an integrative review. Developmental Neuropsychology, 35, 129-155.

Hajcak, G., \& Nieuwenhuis, S. (2006). Reappraisal modulates the electrocortical response to unpleasant pictures. Cognitive, Affective, \& Behavioral Neuroscience, 6, 291-297.

Hajcak G. McDonald N. Simons R.F. (2003). To err is autonomic: errorrelated brain potentials, ANS activity, and post-error compensatory behavior. Psychophysiology, 40, 895-903.

Holroyd, C. B., \& Coles, M. G. (2002). The neural basis of human error processing: reinforcement learning, dopamine, and the error-related negativity. Psychological Review, 109, 679-709.

Inzlicht, M., Bartholow, B. D., \& Hirsh, J. B. (2015). Emotional foundations of cognitive control. Trends in Cognitive Sciences, 19, 126-132.

Lang, P., Bradley, M., \& Cuthbert, B. (2008). International Affective Picture System (IAPS): Affective ratings of pictures and instruction manual. Gainesville: University of Florida.

Liu, Y., Huang, H., McGinnis-Deweese, M., Keil, A., \& Ding, M. (2012). Neural substrate of the late positive potential in emotional processing. Journal of Neuroscience, 32, 14563-14572.

Mischkowski, D., Crocker, J., \& Way, B. M. (2016). From painkiller to empathy killer: acetaminophen (paracetamol) reduces empathy for pain. Social Cognitive and Affective Neuroscience, 11, 1345-1353.

Mischkowski, D., Crocker, J., \& Way, B. M. (2019). A social analgesic? Acetaminophen (Paracetamol) reduces positive empathy. Frontiers in Psychology, 10:538. doi: https://doi.org/10.3389/fpsyg.2019.00538

Mutti, S., Samayoa, J. G., Kam, J., Randles, D., Heine, S., \& Handy, T. C. (2019). The influence of acetaminophen on task related attention. Frontiers in Neuroscience, 13, 444. doi:https://doi.org/10.3389/ fnins.2019.00444

Nieuwenhuis, S., Ridderinkhof, K. R., Blom, J., Band, G. P., \& Kok, A. (2001). Error-related brain potentials are differentially related to awareness of response errors: evidence from an antisaccade task. Psychophysiology, 38, 752-760.

Oldfield, R.C. (1971). The assessment and analysis of handedness: the Edinburgh inventory. Neuropsychologia. 9, 97-113. 
Potts, G. F., Martin, L. E., Kamp, S.-M., \& Donchin, E. (2011). Neural responses to action and reward prediction errors: Comparing the error related negativity to behavioral errors and the feedback related negativity to reward prediction violations. Psychophysiology, 48, 218-228.

Pickering, G., Kastler, A., Macian, N., Pereira, B., Valabregue, R., Lehericy, S., ... \& Jean, B. (2015). The brain signature of paracetamol in healthy volunteers: a double-blind randomized trial. Drug Design, Development and Therapy, 9, 3853-3862.

Proudfit, G. H. (2015). The reward positivity: From basic research on reward to a biomarker for depression. Psychophysiology, 52, 449-459.

Ratner, K. G., Kaczmarek, A. R., \& Hong, Y. (2018). Can Over-theCounter Pain Medications Influence Our Thoughts and Emotions?. Policy Insights from the Behavioral and Brain Sciences, 5, 82-89.

Randles D., Heine S. J., \& Santos, N. (2013). The common pain of surrealism and death: Acetaminophen reduces compensatory affirmation following meaning threats. Psychological Science, 24, 966-973.

Randles, D., Kam, J. W. Y., Heine, S. J., Inzlicht, M., \& Handy, T. C. (2016). Acetaminophen attenuate error evaluation in cortex. Social Cognitive and Affective Neuroscience, 11, 899-906.

Sabatinelli, D., Lang, P. J., Keil, A., \& Bradley, M. M. (2006). Emotional perception: correlation of functional MRI and event-related potentials. Cerebral Cortex, 17, 1085-1091.

Sabatinelli, D., Keil, A., Frank, D. W., \& Lang, P. J. (2013). Emotional perception: correspondence of early and late event-related potentials with cortical and subcortical functional MRI. Biological Psychology, 92, 513-519.

Schmid, P. C., Hackel, L. M., Jasperse, L., \& Amodio, D. M. (2018). Frontal cortical effects on feedback processing and reinforcement learning: Relation of EEG asymmetry with the feedback-related negativity and behavior. Psychophysiology, 55, e12911.

Schupp, H. T., Cuthbert, B. N., Bradley, M. M., Cacioppo, J. T., Ito, T., \& Lang, P. J. (2000). Affective picture processing: The late positive potential is modulated by motivational relevance. Psychophysiology, 37, 257-261.

Schupp, H. T., Junghöfer, M., Weike, A. I., \& Hamm, A. O. (2004). The selective processing of briefly presented affective pictures: an ERP analysis. Psychophysiology, 41, 441-449.

Semlitsch, H. V., Anderer, P., Schuster, P., \& Presslic (1986). A solution for reliable and valid reduction of ocular artifacts, applied to the P300 ERP. Psychophysiology, 23, 695-703.

Smith, H. S. (2009). Potential analgesic mechanisms of acetaminophen. Pain Physician, 12, 269-280.

Spunt, R. P., Lieberman, M. D., Cohen, J. R., \& Eisenberger, N. I. (2012). The phenomenology of error processing: the dorsal ACC response to stop-signal errors tracks reports of negative affect. Journal of Cognitive Neuroscience, 24, 1753-1765.

Thigpen, N. N., Kappenman, E. S., \& Keil, A. (2017). Assessing the internal consistency of the event-related potential: An example analysis. Psychophysiology, 54, 123-138.

Toussaint, K., Yang, X. C., Zielinski, M. A., Reigle, K. L., Sacavage, S. D., Nagar, S., \& Raffa, R. B. (2010). What do we (not) know about how paracetamol (acetaminophen) works?. Journal of Clinical Pharmacy and Therapeutics, 35, 617-638.

Watson, D., \& Clark, L. A. (1999). The PANAS-X: Manual for the Positive and Negative Affect Schedule-Expanded Form. The University of Iowa.

Yeung, N., Holroyd, C. B., \& Cohen, J. D. (2004). ERP correlates of feedback and reward processing in the presence and absence of response choice. Cerebral Cortex, 15, 535-544.

Yoon, E., Babar, A., Choudhary, M., Kutner, M., \& Pyrsopoulos, N. (2016). Acetaminophen-induced hepatotoxicity: A comprehensive update. Journal of Clinical and Translational Hepatology, 4, 131-142.

Publisher's note Springer Nature remains neutral with regard to jurisdictional claims in published maps and institutional affiliations. 